\title{
Термоэлектрические свойства моносилицида кобальта и сплавов на его основе
}

\author{
(C) А.С. Антонов ${ }^{1,2}$, С.В. Новиков ${ }^{1}$, Д.А. Пшенай-Северин ${ }^{1,2}$, А.Т. Бурков ${ }^{1}$ \\ ${ }^{1}$ Физико-технический институт им. А.Ф. Иоффре Российской академии наук, \\ 194021 Санкт-Петербург, Россия \\ ${ }^{2}$ Санкт-Петербургский политехнический университет Петра Великого, \\ 195251 Санкт-Петербург, Россия \\ E-mail: antonov.arts@gmail.com
}

(Поступила в Редакцию 20 декабря 2018 г.

В окончательной редакции 24 декабря 2018 г.

Принята к публикации 28 декабря 2018 г.)

\begin{abstract}
Исследованы термоэлектрические свойства образцов моносилицида кобальта и сплавов на его основе с замещением кобальта железом или никелем. Исследовался диапазон составов с содержанием железа до 10 ат\% и никеля до 5 ат\%. В работе измерены коэффициент термоэдс и удельное сопротивление при температурах 100-800 K. Недавние расчеты зонной структуры моносилицида кобальта выявили ее существенные отличия от простой полуметаллической модели с энергетическим перекрытием параболических зон электронов и дырок. Это потребовало модификации использованных ранее методов описания транспортных свойств. Мы проанализировали возможность интерпретации полученных температурных и концентрационных зависимостей термоэдс и электросопротивления с использованием различных моделей описания электронного спектра.
\end{abstract}

DOI: 10.21883/FTP.2019.05.47561.19

\section{1. Введение}

Изучение термоэлектрических свойств моносилицида кобальта и его твердых растворов с FeSi и $\mathrm{NiSi}$ было начато в работах [1-3]. Данные материалы привлекают внимание тем, что обладают хорошими механическими характеристиками, устойчивы при высоких температуpax, не содержат дорогих или токсичных компонентов и имеют достаточно высокие для полуметаллов значения коэффициента термоэдс [1-3]. Материал имеет кубическую кристаллическую структуру (тип В20, пространственная группа $\left.P 2{ }_{1} 3-T^{4}\right)$. В элементарной ячейке (с параметром $a=4.449 \AA$ ) содержится по 4 атома кремния и кобальта $[2,3]$.

Концентрация электронов в моносилициде кобальта может быть изменена путем создания его твердых растворов с NiSi или FeSi. FeSi и CoSi формируют непрерывную серию твердых растворов, а изоструктурные твердые растворы $\mathrm{Co}_{1-x} \mathrm{Ni}_{x} \mathrm{Si}$ образуются при содержании $\mathrm{Ni}$ до 10 ат\%. Концентрация электронов увеличивается при замене атомов кобальта атомами никеля и уменьшается при замене атомов кобальта атомами железа [1].

Повышение термоэлектрической эффективности материала требует правильного представления об энергетическом спектре носителей тока. Первые исследования зонной структуры проводились на основе простого описания зонной структуры в виде двух зон (электронной и дырочной) со стандартным (изотропным параболическим) законом дисперсии. В работах $[1,4]$ для анализа зонной структуры исследовались оптические и кинетические свойства материала. Для описания термоэлектрических свойств моносилицида кобаль- та имеется несколько моделей с различными наборами параметров. Свойства твердых растворов на основе $\mathrm{CoSi}$ можно понять, используя модель „жесткой зоны“. Согласно этой модели, твердые растворы $\mathrm{Co}_{1-x} \mathrm{Ni}_{x} \mathrm{Si}$ и $\mathrm{Co}_{1-x} \mathrm{Fe}_{x} \mathrm{Si}$ имеют схожие зонные структуры. С изменением состава меняется только положение уровня Ферми [2]. В ряде недавних исследований [5,6] был проведен расчет зонной структуры $\mathrm{CoSi}$ из первых принципов. Данные исследования показали наличие особенностей спектра, которые не учитываются в простой модели, применяемой для описания полуметаллов. Более того, эти исследования показали, что CoSi относится к классу веществ с нетривиальной топологией зонной структуры. Это потребовало модификации использованных ранее методов описания транспортных свойств.

В настоящей работе получены экспериментальные данные по кинетическим коэффициентам. Проведено сравнение полученных данных с имеющимися в литературе и осуществлен их анализ в рамках теоретических моделей, описанных в [1,4], а также с учетом энергетического спектра электронов, рассчитанного из первых принципов с использованием метода функционала электронной плотности.

\section{2. Техника эксперимента}

В работе исследовались образцы моносилицида кобальта и его твердых растворов с FeSi и NiSi в диапазоне составов с содержанием железа до 10 ат\% и никеля до 5 ат\%. Материалы для получения образцов были синтезированы методом прямого сплавления. Навески 
материалов сплавляли в индукционной печи. После охлаждения материалы были дополнительно протянуты по методу Бриджмена для рекристаллизации. Порошковая рентгенография показала, что полученные образцы являются однофазными и постоянная решетки меняется линейно с изменением состава.

Для измерения термоэдс был применен дифференциальный метод, а для измерения электропроводности четырехзондовый метод. Описание установки, реализующей данные методы измерений в исследуемом диапазоне температур, представлено в [7,8].

\section{3. Расчет кинетических коэффициентов}

Для расчета кинетических коэффициентов были использованы две теоретических модели описания энергетического спектра носителей заряда в материале.

Первая модель основывается на простом описании зонной структуры в виде двух зон (электронной и дырочной) с изотропным параболическим законом дисперсии. В работах $[1,4]$ проводились исследования моносилицида кобальта и его твердых растворов. Для интерпретации и анализа температурных зависимостей в этих работах использовалась упомянутая выше модель электронного спектра и учитывалось рассеяние носителей заряда на акустических фононах и точечных дефектах. Время релаксации для рассеяния на близкодействующем потенциале точечных дефектов имеет ту же энергетическую зависимость, что и для акустического рассеяния, но не зависит явно от температуры $T$. Зависимость времени релаксации в этом случае определяется выражением $\tau^{-1} \propto\left(T / T_{0}+w\right) \sqrt{\varepsilon}$, где $T_{0}=300 \mathrm{~K}$, а постоянная $w$ определяет относительную интенсивность рассеяния на дефектах по сравнению с акустическим рассеянием. При этом подвижности электронов и дырок задаются выражениями

$$
u_{n(p)}=\frac{u_{0 n, 0 p}}{\left(T / T_{0}+w\right) \sqrt{T / T_{0}}} \frac{F_{0}\left(\mu_{n, p}^{*}\right)}{F_{1 / 2}\left(\mu_{n, p}^{*}\right)},
$$

где $\mu_{n, p}^{*}-$ химический потенциал электронов (дырок) в единицах $k_{0} T$, интегралы Ферми $F_{v}\left(\mu_{n, p}^{*}\right)=$ $=\int_{0}^{\infty} f_{0}\left(x, \mu_{n, p}^{*}\right) x^{v} d x, f_{0}\left(x, \mu_{n, p}^{*}\right)-$ функция распределения Ферми.

В модели [4] предполагалось, что величина $b=u_{0 n} / u_{0 p}$, определяющая отношение подвижностей электронов и дырок, не зависит от температуры. Предполагается также, что разность концентраций электронов и дырок $\Delta N$ и эффективные массы плотности состояний $m_{d n, d p}^{*}$ не зависят от температуры. Учитывалась линейная зависимость энергии перекрытия валентной зоны и зоны проводимости от температуры $\Delta E=\Delta E_{0}+\beta T[4]$.
Концентрации электронов $(n)$ и дырок $(p)$ задаются выражениями

$$
n, p=4 \pi\left(\frac{2 m_{d n, d p}^{*} k_{0} T}{h^{2}}\right)^{3 / 2} F_{1 / 2}\left(\mu_{n, p}^{*}\right) .
$$

Химические потенциалы $\mu_{n, p}^{*}$ определялись из условия $\Delta N=n-p$ с учетом соотношения $\mu_{n}^{*}+\mu_{p}^{*}=\Delta E / k_{0} T$. Термоэдс может быть вычислена по формуле

$$
S=\frac{S_{p} \sigma_{p}-S_{n} \sigma_{n}}{\sigma_{p}+\sigma_{n}}
$$

где термоэдс электронов (индекс $n)$ и дырок (индекс $p$ ) определяются выражениями

$$
S_{n, p}=\frac{k_{0}}{e}\left(2 \frac{F_{1}\left(\mu_{n, p}^{*}\right)}{F_{0}\left(\mu_{n, p}^{*}\right)}-\mu_{n, p}^{*}\right) .
$$

Из формул (1)-(4) видно, что термоэдс кроме температуры зависит еще от 6 параметров: от эффективных масс $\left(m_{d n}^{*}, m_{d p}^{*}\right)$, разности концентраций $(\Delta N)$, отношения подвижностей носителей заряда $b$, величины энергетического зазора при нулевой температуре $\left(\Delta E_{0}\right)$ и ее температурного коэффициента $(\beta)[4]$. В рамках данной модели при анализе экспериментальных зависимостей проводится подбор этих 6 параметров таким образом, чтобы добиться наилучшего согласия теоретически рассчитанных зависимостей термоэдс и электропроводности с экспериментальными.

Вторая модель основана на результатах расчета энергетического спектра по методу функционала электронной плотности в пакете QuantumEspresso [9]. Расчет проводился с использованием градиентного приближения для функционала электронной плотности без учета спин-орбитального взаимодействия при равновесных параметрах решетки (параметр решетки $a=4.4348 \AA$, доля кобальта $x_{\mathrm{Co}}=0.144$, доля кремния $x_{\mathrm{Si}}=0.843$ ). Число плоских волн определялось граничной энергией 90Ry, a интегрирование по зоне Бриллюэна проводилось на сетке Монхорста-Пака $6 \times 6 \times 6$. Картина зонной структуры и плотность состояний, полученные в результате расчета в данной работе (рис. 1), хорошо согласуются с приведенными в литературе [5,6]. В рамках данной модели кинетические коэффициенты могут быть рассчитаны с помощью формул, описанных в [10]. При этом для вычисления кинетических коэффициентов необходимо использовать достаточно плотную сетку в зоне Бриллюэна $(64 \times 64 \times 64)$, что было сделано с использованием Ваннье-интерполяции зонной структуры [10] и потребовало проведения дополнительных расчетов по сравнению с работами $[5,6]$.

Влияние изменения состава в данной модели учитывается через сдвиг химического потенциала $\mu$ в приближении ,жестких зон“.

В рамках данной модели кинетические коэффициенты термоэдс $S$ и электропроводности $\sigma$ могут быть найдены 

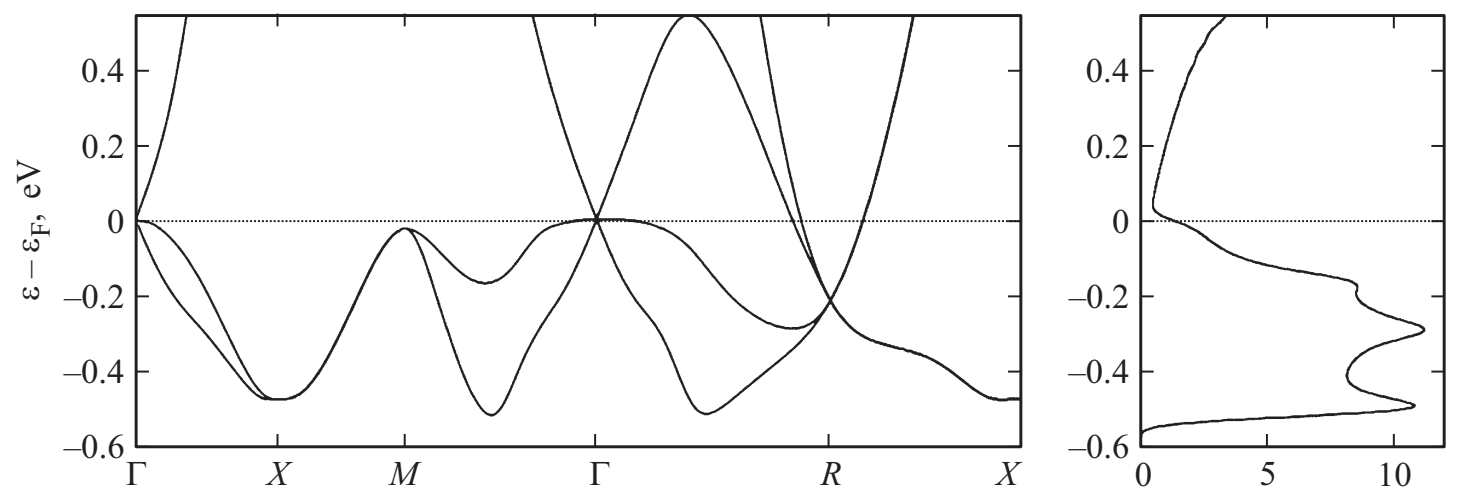

DOS, states/eV/unit cell

Рис. 1. Электронная зонная структура и плотность состояний (DOS) в $\mathrm{CoSi}$, рассчитанная по методу функционала электронной плотности.
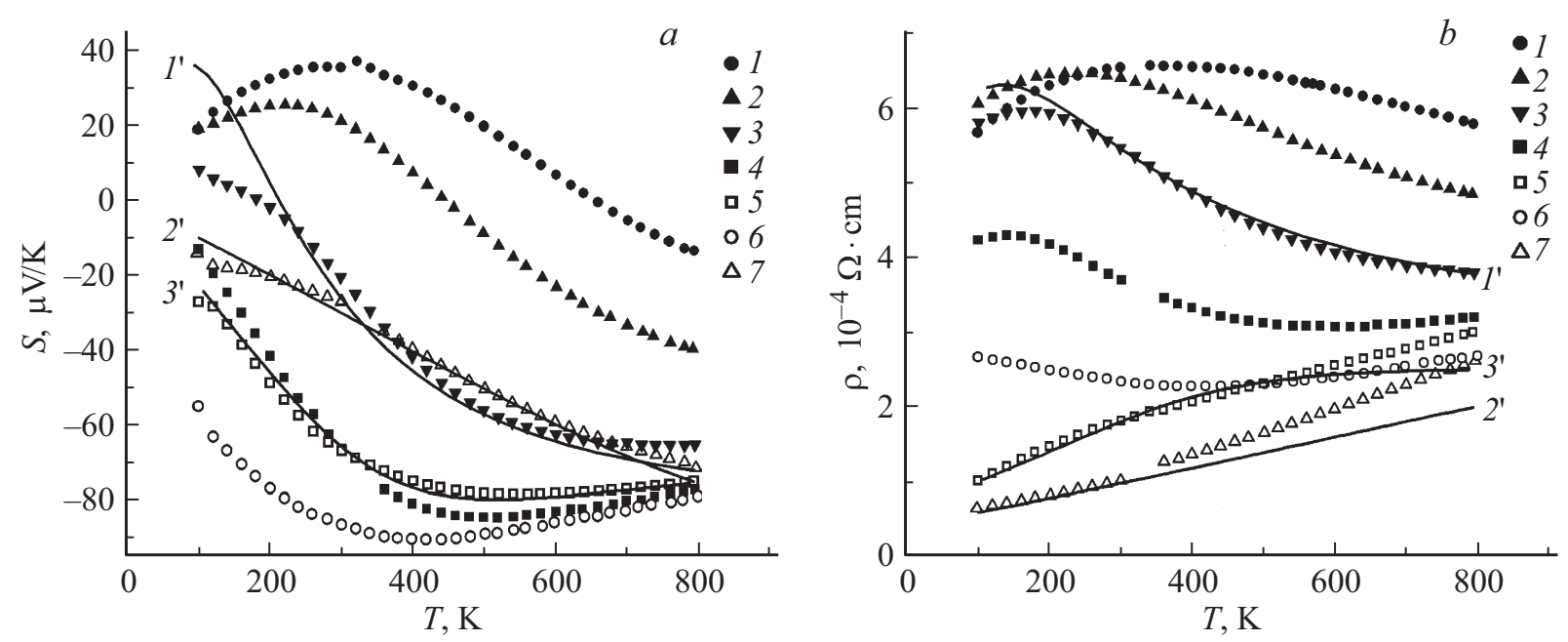

Рис. 2. Температурные зависимости термоэдс $S(a)$ и удельного сопротивления $\rho(b)$. Экспериментальные данные (точки): $1-\mathrm{Co}_{0.9} \mathrm{Fe}_{0.1} \mathrm{Si}, 2-\mathrm{Co}_{0.92} \mathrm{Fe}_{0.08} \mathrm{Si}, 3-\mathrm{Co}_{0.96} \mathrm{Fe}_{0.04} \mathrm{Si}, 4-\mathrm{Co}_{0.985} \mathrm{Fe}_{0.015} \mathrm{Si}, 5-\mathrm{CoSi}, 6-\mathrm{Co}_{0.98} \mathrm{Fe}_{0.01} \mathrm{Ni}_{0.01} \mathrm{Si}_{2} 7-\mathrm{Co}_{0.95} \mathrm{Ni}_{0.05} \mathrm{Si}$. Теоретические зависимости (сплошная линия): $1^{\prime}-\mathrm{Co}_{0.96} \mathrm{Fe}_{0.04} \mathrm{Si}, 2^{\prime}-\mathrm{Co}_{0.95} \mathrm{Ni}_{0.05} \mathrm{Si}, 3^{\prime}-\mathrm{CoSi}$.

на основе следующих выражений [10]:

$$
\begin{gathered}
{[\sigma]_{i j}(\mu, T)=e^{2} \int_{-\infty}^{+\infty} d \varepsilon\left(-\frac{d f_{0}\left(\varepsilon / k_{0} T, \mu^{*}\right)}{d \varepsilon}\right) \Sigma_{i j}(\varepsilon),} \\
{[\sigma S]_{i j}(\mu, T)=\frac{e}{T} \int_{-\infty}^{+\infty} d \varepsilon\left(-\frac{d f_{0}\left(\varepsilon / k_{0} T, \mu^{*}\right)}{d \varepsilon}\right)(\varepsilon-\mu) \Sigma_{i j}(\varepsilon),}
\end{gathered}
$$

где $\Sigma_{i j}(\varepsilon)$ - тензор транспортной функции распределения

$$
\boldsymbol{\Sigma}_{i j}(\varepsilon)=\frac{1}{V} \Sigma_{n, \mathbf{k}} v_{i}(n, \mathbf{k}) v_{j}(n, \mathbf{k}) \tau(n, \mathbf{k}) \delta\left(\varepsilon-E_{n, \mathbf{k}}\right) .
$$

В рассматриваемой модели были использованы два приближения для времени релаксации: приближение постоянного времени релаксации (CRTA) и приближение $\tau^{-1} \propto\left(T / T_{0}+w\right) g(\varepsilon)$, учитывающее рассеяние электронов на фононах и точечных дефектах. В данном выражении постоянная $w$ определяет относительную интенсивность рассеяния на дефектах, а $g(\varepsilon)-$ плотность состояний. При этом расчет с использованием $\tau(\varepsilon)$ потребовал разработки собственной программы для расчета кинетических коэффициентов. В твердых растворах с замещением кобальта железом или никелем концентрация дефектов пропорциональна доле замещаемых атомов, поэтому $w=w_{0} x / x_{0}$, где $w_{0}$ не зависит от состава, а $x_{0}=0.04$.

\section{4. Результаты и их обсуждение}

Для всех образцов были получены температурные зависимости термоэдс $S$ и удельного сопротивления $\rho$ в диапазоне $100-800 \mathrm{~K}$. Данные зависимости представлены на рис. 2, $a$ и $b$ соответственно.

Была проанализирована возможность интерпретации полученных температурных и концентрационных зави- 
Параметры твердых растворов на основе моносилицида кобальта

\begin{tabular}{c|c|c|c|c|c|c|c}
\hline Состав & $\Delta E$, мэB & $\beta, 10^{-6}{ }_{\ni} \mathrm{B} / \mathrm{K}$ & $\Delta N, 10^{20} \mathrm{~cm}^{-3}$ & $m_{d n} / m_{e}$ & $m_{d p} / m_{e}$ & $b$ & $w$ \\
\hline $\mathrm{CoSi}$ & -20 & 65 & 4.5 & 2 & 6 & 5.4 & 0.55 \\
$\mathrm{Co}_{0.95} \mathrm{Ni}_{0.05} \mathrm{Si}$ & -35 & 26 & 15.6 & 2 & 6 & 8 & 0.68 \\
$\mathrm{Co}_{0.96} \mathrm{Fe}_{0.04} \mathrm{Si}$ & 20 & 48 & -5 & 2 & 6 & 8.6 & 0.84
\end{tabular}

симостей с использованием вышеописанных моделей описания электронного спектра. На основе первой модели осуществлен подбор параметров, обеспечивающих качественное согласование теоретических и полученных экспериментальных зависимостей. Подобранные параметры модели для ряда образцов приведены в таблице, а теоретические зависимости $S(T)$ и $\rho(T)$, рассчитанные с подобранным набором параметров, представлены на рис. 2. Как было сказано выше, для образцов твердых растворов необходимо скорректировать эту зависимость с учетом рассеяния на близкодействующем потенциале точечных дефектов. Температурная зависимость электропроводности для образца моносилицида кобальта должна хорошо описываться только с учетом акустического рассеяния, т.е. при $w=0$. В данной работе для $\mathrm{CoSi}$ параметр оказался равным $w=0.55$, что, по-видимому, обусловлено присутствием в образце собственных точечных дефектов, не связанных с добавками железа или никеля.

В результате сравнения экспериментальных данных, полученных в настоящей работе и в работах $[1,4]$, выявлено их значительное несоответствие. Хотя теоретические зависимости, рассчитанные с использованием первой модели, качественно описывают экспериментальные зависимости, однако из-за большого количества параметров их определение путем подгонки к экспериментальным зависимостям неоднозначно. Поэтому представляет интерес использование расчетных данных об энергетическом спектре носителей для расчета свойств.

На рис. 3 приведены температурные зависимости термоэдс и электропроводности для чистого моносилицида кобальта, полученные с использованием расчетного спектра, в сравнении с экспериментальными данными. На рис. 4, $a$ и $b$ приведены зависимости тех же кинетических коэффициентов от состава при комнатной температуре. В обоих вариантах модели расчет термоэдс не содержит подгоночных параметров. Единственный подгоночный параметр появляется при вычислении электропроводности и определяется ее абсолютной величиной. Из рисунков видно, что результаты расчетов, полученные с использованием приближения постоянного времени релаксации, не позволяют описать экспериментальные температурные и концентрационные зависимости: в этом приближении не удается получить даже верный знак коэффициента термоэдс. В то же время учет энергетической зависимости времени релаксации $\tau(\varepsilon)$ позволяет получить качественное согласие теоретических и экспериментальных зависимостей.

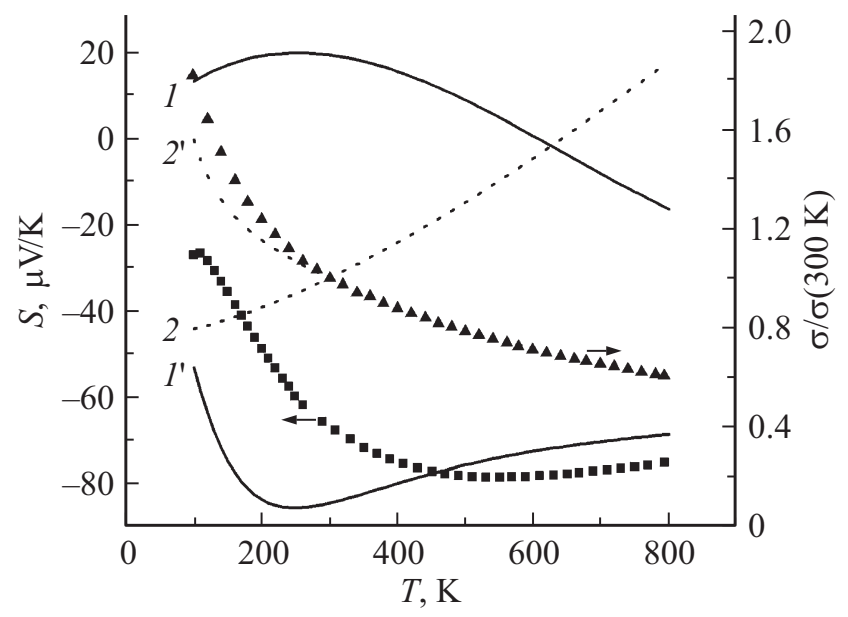

Рис. 3. Температурные зависимости термоэдс $S$ и электропроводности $\sigma / \sigma(300 \mathrm{~K})$ для $\mathrm{CoSi}$. Точки - эксперимент, сплошные линии - теоретический расчет в приближениях CRTA $(1,2)$ и $\tau(\varepsilon)\left(1^{\prime}, 2^{\prime}\right)$ для термоэдс $\left(1,1^{\prime}\right)$ и электропроводности $\left(2,2^{\prime}\right)$.

Полученное согласие с экспериментом при использовании для расчета времени релаксации полной плотности состояний говорит о том, что в данных материалах заметный вклад в рассеяние вносят межзонные переходы. Плотность состояний сильно возрастает при уменьшении энергии в области $\varepsilon<\varepsilon_{\mathrm{F}}$, а ее рост при $\varepsilon>\varepsilon_{\mathrm{F}}$ оказывается более слабым (см. рис. 1). Это приводит к тому, что по сравнению с приближением CRTA вклад в термоэдс электронов вблизи точки $R$ зоны Бриллюэна возрастает, а вклад дырок вблизи точек $\Gamma$ и $M$ снижается. В результате термоэдс оказывается отрицательной, в согласии с экспериментом (рис. 4, a).

Используемая энергетическая зависимость времени релаксации позволяет также объяснить асимметрию зависимости электропроводности от состава (рис. 4, $b$ ). В твердых растворах CoSi с железом электропроводность оказывается ниже, чем при добавлении никеля, и уменьшается с ростом содержания железа. При использовании постоянного времени релаксации (рис. $4, b$, сплошная кривая) проводимость возрастает как при добавлении железа, так и при добавлении никеля, что связано с увеличением концентрации дырок и электронов соответственно. С учетом энергетической зависимости времени релаксации оказывается, что в твердых растворах $\mathrm{CoSi}$ с железом химический потенциал смещается в область более низких энергий и более 

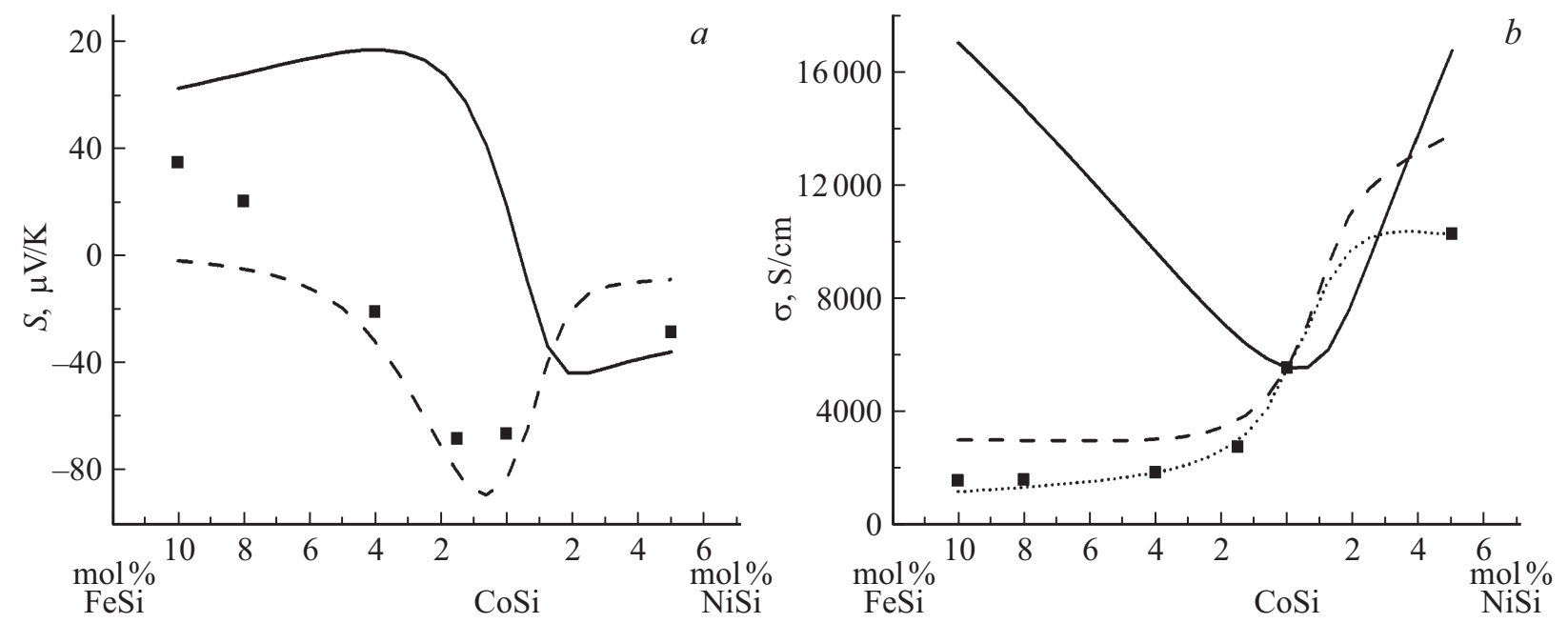

Рис. 4. Термоэдс $S(a)$ и электропроводность $\sigma(b)$ в зависимости от состава твердого раствора при $300 \mathrm{~K}$. Точки - эксперимент, сплошные линии - теоретический расчет в приближении CRTA (сплошная линия), а также в приближении $\tau(\varepsilon)$ с учетом рассеяния на примесных дефектах (штриховая) и без его учета (пунктирная).

высокой плотности состояний, в которой вероятность рассеяния носителей возрастает, а подвижность снижается. На рис. 4, $b$ пунктирная кривая построена с учетом рассеяния только на фононах. Уже для этого случая видно, что зависимость от состава качественно повторяет экспериментальную. Учет дополнительного рассеяния на дефектах в твердом растворе (рис. $4, b$, точечная кривая) позволяет получить лучшее согласие с экспериментом при значениях $w_{0}=0.28$ для $\mathrm{Co}_{1-x} \mathrm{Ni}_{x} \mathrm{Si}$ и $w_{0}=0.63$ для $\mathrm{Co}_{1-x} \mathrm{Fe}_{x} \mathrm{Si}$. Таким образом, электропроводность в твердых растворах с $\mathrm{FeSi}$ оказывается ниже, чем в чистом $\mathrm{CoSi}$ и твердых растворах с $\mathrm{NiSi}$, в согласии с экспериментом, за счет большей плотности состояний на уровне химического потенциала и большей интенсивности рассеяния на дефектах.

\section{5. Заключение}

В результате сравнения модельных расчетов кинетических коэффициентов с экспериментальными данными установлено, что простая двухзонная модель в принципе может описать термоэлектрические свойства, но зависит от большого числа параметров, выбор которых может быть неоднозначным.

Сравнение температурных зависимостей электропроводности чистого CoSi и твердых растворов с заменой кобальта на $\mathrm{Ni}$ или $\mathrm{Fe}$ показало, что в последних заметную роль играет рассеяние на точечных дефектах. Вклад этого механизма рассеяния оказывается более сильным для твердых растворов $\mathrm{Co}_{x} \mathrm{Fe}_{1-x} \mathrm{Si}$.

Вычисление термоэдс и электросопротивления с использованием электронного спектра, рассчитанного из первых принципов, в приближении постоянного времени релаксации не позволяет даже качественно учесть зависимости кинетических коэффициентов от температуры и состава. При учете энергетической зависимости времени релаксации первопринципный расчет позволяет качественно и в значительной степени количественно, описать основные особенности зависимостей кинетических коэффициентов от температуры и состава. Для термоэдс расчет не содержит подгоночных параметров. Единственный подгоночный параметр появляется при вычислении электропроводности (определяется ее абсолютной величиной).

Хорошее согласие с экспериментом в предположении, что $\tau$ обратно пропорционально плотности состояний, указывает на важную роль межзонного рассеяния. В частности, наряду с положением уровня химического потенциала, оно определяет знак термоэдс. Кроме того, учет $\tau(\varepsilon)$ позволяет объяснить асимметрию зависимости сопротивления от состава твердых растворов.

\section{Список литературы}

[1] S. Asanabe, D. Shinoda, Y. Sasaki. Phys. Rev., 134 (3A), A774 (1964).

[2] П.В. Гельд, Ф.А. Сидоренко. Силициды переходных металлов четвертого периода (М., Металлургия, 1971).

[3] M.I. Fedorov, V.K. Zaitsev. In: CRC Handbook of Thermoelectrics, ed. by D.M. Rowe (1995) p. 321.

[4] V.K. Zaitsev, L.S. Stilbans, V.I. Tarasov, V.I. Fedorov, N.V. Kolomoets. Proc. II Int. Conf. on Thermoelectrical Energy Conversion (Arlington, 1978) p. 23.

[5] A. Sakai, F. Ishii, Y. Onose. J. Phys. Soc. Jpn., 76 (9), 1 (2007).

[6] D.A. Pshenay-Severin, Yu.V. Ivanov, A.A. Burkov, A.T. Burkov. J. Phys.: Condens. Matter., 30, 135501 (2018).

[7] А.Т. Бурков, А.И. Федотов, А.А. Касьянов, Р.И . Пантелеев, Т. Накама. Науч.-техн. вестн. информационных технологий, механики и оптики, 15 (2), 173 (2015).

[8] A.T. Burkov, A. Heinrich, P.P. Konstantinov, T. Nakama, K. Yagasaki. Meas. Sci. Technol., 12, 264 (2001). 
[9] P. Giannozzi, O. Andreussi, T. Brumme, O. Bunau, M.B. Nardelli, M. Calandra. J. Phys.: Condens. Matter, 29 465901 (2017).

[10] G. Pizzi, D. Volja, B. Kozinsky, M. Fornari, N. Marzaru. Comp. Phys. Commun., 185, 422 (2014).

Редактор Л.В. Шаронова

\section{Thermoelectric properties of cobalt monosilicide and alloys based on it}

A.S. Antonov ${ }^{1,2}$, S.V. Novikov ${ }^{1}$, D.A. Pshenay-Severin ${ }^{1,2}$, A.T. Burkov ${ }^{1}$

${ }^{1}$ loffe Institute,

Russian Academy of Sciences, 194021 St. Petersburg, Russia

${ }^{2}$ Peter the Great St.Petersburg Polytechnic University, 195251 St. Petersburg, Russia

Abstract The samples of cobalt monosilicide CoSi and its alloys with the substitution of iron or nickel for cobalt $\left(\mathrm{Co}_{1-x} \mathrm{M}_{x} \mathrm{Si}\right.$, $\mathrm{M}=\mathrm{Fe}, \mathrm{Ni}$ ) were studied. The investigations were made for alloy compositions with iron content up to 10 at $\%$ and nickel up to $5 \mathrm{at} \%$. The thermopower and electrical resistivity were measured in the temperature range of $100-800 \mathrm{~K}$. Recent calculations of the cobalt monosilicide band structure revealed a number of essential differences from the standard semi-metallic model with energy overlap of parabolic bands for electrons and holes. This raises the question on the effect of the new band structure features on the theoretical interpretation of experimental date of the compound. We analyze the possibility of theoretical interpretation of temperature and concentration dependences of the thermopower and electrical resistivity using different models of the electron spectrum. 